\title{
East Meets West: Synergy through Diversity
}

\author{
Stefan Hager ${ }^{a}$ Jingzhang Dai ${ }^{a, b}$ Volker Fischer $^{a}$ Folkert Lüthke ${ }^{a}$ Anton Staudinger ${ }^{a}$ \\ a TCM Hospital Bad Kötzting, Bad Kötzting, Germany \\ ${ }^{\mathrm{b}}$ Beijing University of Chinese Medicine, Beijing, China
}

\section{Keywords}

Traditional Chinese Medicine .

TCM hospital Bad Kötzting · Psychosomatic medicine ·

Lifestyle medicine $\cdot$ Psychoeducation

\section{Summary}

The TCM hospital Bad Kötzting is historically the first inpatient facility in Germany with an emphasis on Traditional Chinese Medicine (TCM). The clinic's specialty is the multimodal treatment of somatic complaints in conjunction with acute psychological or psychiatric comorbidity. Most patients present with clinical problems of a high degree of chronicity and complexity. Over the past 25 years the treatment concept of the hospital has developed from a strictly TCM approach to a multimodal combination of TCM, psychosomatic medicine, and lifestyle medicine. This article outlines the admission and intake procedures and describes the process of determining the TCM diagnoses and treatment protocols. A typical case study illustrates this process in more details. Then, we present the various components of the psychotherapeutic and psychoeducational programs, including innovative approaches to lifestyle medicine. In conclusion, the treatment program at the TCM hospital Bad Kötzting has developed into a multimodal approach that synergistically intertwines diverse therapies drawn from Eastern and Western traditions, effectively combining the best approaches of both contexts.

(C) 2016 S. Karger GmbH, Freiburg

\section{Basic Information}

The TCM hospital Bad Kötzting is historically the first inpatient facility in Germany with an emphasis on Traditional Chinese Medicine (TCM) as the main treatment modality. It is a govern-

\author{
Schlüsselwörter \\ Traditionelle Chinesische Medizin . \\ TCM-Klinik Bad Kötzting · Psychosomatik · \\ TCM · Lebensstilmedizin · Psychoedukation
}

\section{Zusammenfassung}

Die TCM-Klinik Bad Kötzting ist historisch das erste deutsche Krankenhaus mit einem Schwerpunkt auf der Traditionellen Chinesischen Medizin (TCM). Die Klinik spezialisiert sich auf die multimodale Behandlung somatischer Beschwerden, die zusammen mit akuten psychischen Erkrankungen auftreten. Die meisten Patienten leiden an komplexen Symptomen mit einer langen Vorgeschichte konventioneller Behandlungen. In der 25-jährigen Geschichte der Klinik hat sich das Behandlungskonzept von einer reinen TCM-Orientierung hin zu einem multimodalen Ansatz entwickelt, der TCM, Psychosomatik und Lebensstilmedizin verbindet. Dieser Artikel beschreibt den Vorauswahl- und Aufnahmeprozess an der Klinik. Außerdem wird der Prozess der TCM-Diagnosefindung und der daraus abgeleiteten Therapieindikationen erklärt und anhand einer Kasuistik illustriert. Es folgt eine Beschreibung der verschiedenen Komponenten des psychotherapeutischen und psychoedukativen Programms, welches auch innovative Ansätze der Lebensstilmedizin umfasst. Zusammenfassend wird festgestellt, dass die TCM-Klinik Bad Kötzting ein multimodales Behandlungskonzept entwickelt hat, das therapeutische Ansätze östlicher und westlicher Provenienz zusammenführt. Die daraus resultierende Synergie hat sich als überaus fruchtbar erwiesen.

ment-licensed 75-bed hospital which provides care for about 1,000 inpatients and more than 2,000 outpatients a year. Over $90 \%$ of the inpatients are fully covered by the statutory health insurance; about $10 \%$ are self-paying patients. In most cases the hospital stay is about 4 weeks.

\section{KARGER \\ Fax +497614520714 \\ Information@Karger.com}

www.karger.com

\section{() 2016 S. Karger GmbH, Freiburg}

$1661-4119 / 16 / 0237-0003 \$ 39.50 / 0$
Stefan Hager, MD

TCM Hospital Bad Kötzting

Ludwigstrasse 2, 93444 Bad Kötzting, Germany

s.hager@tcm.info 
Fig. 1. Conceptual framework.

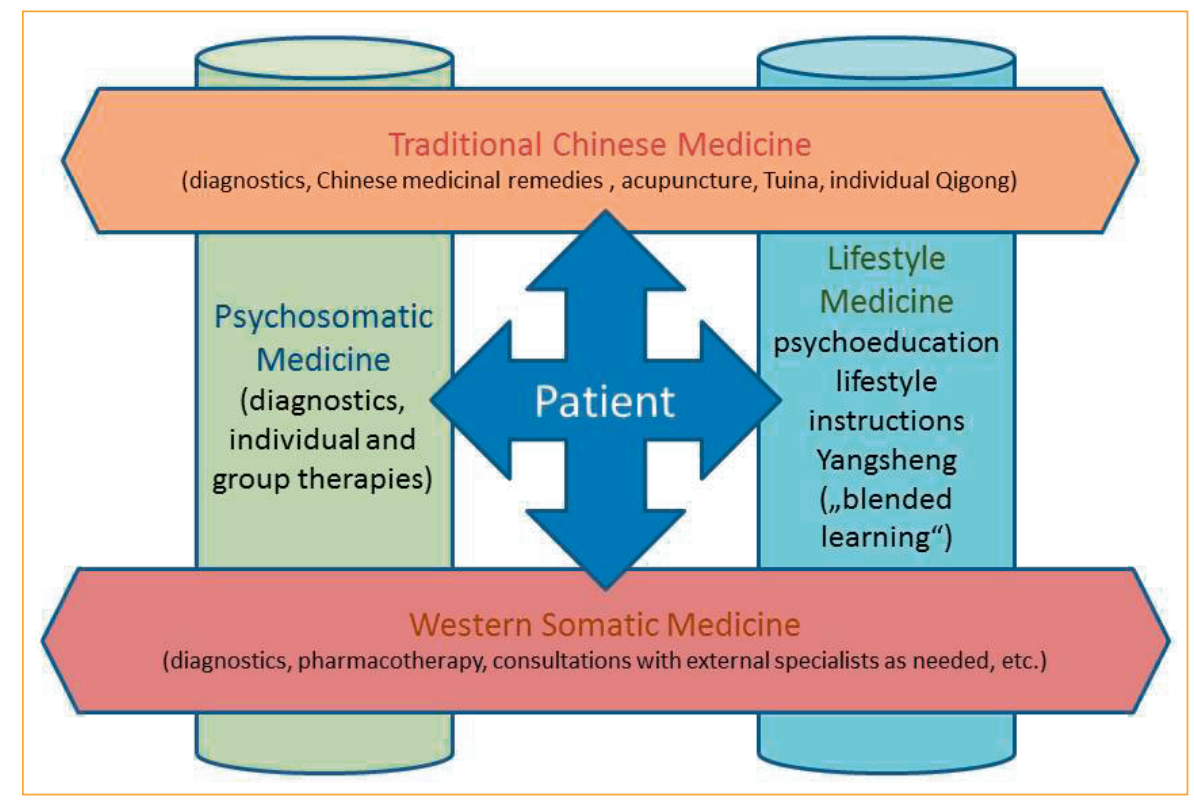

Over the past 25 years the treatment concept has developed from a strictly TCM approach to a multimodal combination of TCM, psychosomatic medicine, and lifestyle medicine (fig. 1).

The majority of our patients present with clinical problems of a high degree of chronicity and complexity. They frequently are referred to us with diagnoses from the neurological, orthopedic, or internal medicine domains. Most come to us after years of conventional treatment have proven unsatisfactory. Essentially, our specialty is the multimodal treatment of somatic complaints in conjunction with acute psychological or psychiatric comorbidity. Thus, the vast majority of our patients carry both, somatic as well as psychiatric diagnoses.

\section{Preadmission Procedures}

As part of our preadmission procedures prospective patients fill out a 4-page questionnaire detailing their physical and psychological complaints, medical history, desired treatment outcome, previous and current diagnoses, findings, etc. On the basis of these data the patient receives one of three responses: (a) the request for treatment is approved and the patient receives information about further proceedings; (b) the request for treatment is denied and the patient receives information about the reasons; (c) a recommendation for outpatient treatment prior to potential inpatient treatment is made.

If a treatment request is accepted, the patient has to procure the assurance of coverage from his or her health insurance carrier. Thereafter, the patient is advised about the prospective date of admission. In addition, patients receive instructions on filling out several internet-based questionnaires prior to admission. These questionnaires are designed to assess various areas of health, stress, coping, resources, and satisfaction with multiple domains of life. The data will be considered as a source of information in the course of the initial medical and psychological assessments.

\section{Patient Admission}

On the day of admission the patient undergoes a first comprehensive interview and examination by a physician trained in Western medicine. In the presence of the patient, the findings from this interview will be jointly presented to a TCM doctor (mediated by a translator) as well as the assigned psychotherapist. The TCM doctor will add the TCM diagnoses based on an evaluation of the history, symptoms, clarifying questions, as well as a tongue and pulse diagnosis. Drawing on this information, a TCM diagnosis and an initial prescription of TCM treatments are being determined. The psychotherapist, in turn, takes the opportunity to gather information about the medical background of the patient and to establish a first rapport.

\section{The TCM Process: Diagnosis and Treatment}

The TCM diagnoses and treatments are performed by doctors and professors of the Bejing University of Chinese Medicine and its associated university hospitals.

The TCM doctor takes a detailed history of physical and vegetative symptoms, augmented by information gained through tongue and pulse diagnosis (signs). Based on this comprehensive picture the TCM doctor arrives at an energetic diagnosis on the basis of the conceptual framework of TCM (taking into consideration concepts like Qi, blood, yin/yang, 6-layer model, etc.). Signs and symptoms will be clustered around uniquely 'Chinese' nosological categories and diagnostic terms, such as stagnation of meridians, liver-Qi stagnation, blood stasis, dampness, etc.

We shall illustrate the TCM diagnostic process for a hypothetical female patient with symptoms of chronic pain and a current diagnosis of moderate depression. This patient has a longstanding history of back-pain; chronic personal and professional stress and 
overwhelm; loss of energy; a 'chordal' pulse, and a bluish tongue with bluish stains.

For a diagnostic workup, the TCM-doctor sorts the observed symptoms and signs into clusters:

Symptom cluster I (muscular tension, 'chordal' pulse) gives rise to the diagnosis of liver-Qi stagnation. According to TCM teachings, the liver network is of primary importance for coping with stress and stimuli. Blockages in the flow of liver-Qi result in blockages in the circulation of bodily fluids, which accounts for the observation of the bluish tongue with bluish stains. Consequently, stasis of the blood gets coded into the diagnostic framework.

Symptom cluster II is comprised of the following signs and symptoms: loss of appetite; fatigue with only $20 \%$ residual energy; dry mouth; night sweats; increased ruminations; difficulty concentrating; burning pain in the thighs; pulse quality 'slippery'. This symptom cluster suggests to the TCM doctor some digestive problems (coded as spleen-Qi blockage) and the resulting deficit in energy and the availability of bodily fluids, coded as spleen-Qi deficiency.

Symptom cluster III includes: loss of interest; anhedonia; insomnia; restlessness and difficulty concentrating. According to TCM concepts, a disturbance in the heart network can lead to impaired consciousness, insomnia, difficulty concentrating, and diminished memory, thus resulting in mental and emotional impairments. Hence, the TCM doctor would diagnose blockages in the flow of heart-Qi.

Symptom cluster IV encompasses the following symptoms and signs: loss of interest; back pain; fatigue; feeling exhausted and 'beaten up' at night; difficulty concentrating; loss of libido; weak 'kidney pulse.' According to the teachings of TCM, low vitality, diminished sex drive, and lower back pain together suggest an impairment of the kidney network, leading to a diagnosis of kidney-Qi deficiency.

In summary, the complete list of TCM diagnoses for this sample patient would read as follows: liver-Qi stagnation with stasis of the blood; spleen-Qi blockage with spleen-Qi deficiency; heart-Qi disturbance, and kidney-Qi deficiency.

\section{General TCM Treatments at the TCM Hospital Bad Kötzting}

The primary TCM treatment modalities include highly concentrated Chinese medicinal remedies, acupuncture, Tuina (massage) and Qigong. Based on the TCM diagnosis, the TCM doctor prescribes a treatment designed to address the observed patterns of symptoms.

First, there will be a prescription for Chinese medicinal remedies, often a mixture of 10-15 herbs amounting to a total of $60-$ $100 \mathrm{~g}$, which will be boiled and turned into approximately $200 \mathrm{ml}$ of a decoction to be taken twice daily.

Second, a prescription for individualized acupuncture treatments will be formulated. Most patients receive three acupuncture treatments per week. Since a vast majority of patients also suffer from pain in their muscles, joints or back, two manual treatments - either Tuina massage or individual Qigong treatment - will be added to the prescribed regimen in most cases.
Furthermore, all patients participate in a series of 18 exercises derived from time-tested Tai Chi-Qigong traditions. The exercises incorporate slow movements, deep breathing techniques, and imagery components. These meditative movements are being modelled and practiced in a group setting for $30 \mathrm{~min}$ in the morning and in the afternoon. Video instructions are also accessible on demand for all patients.

In addition, all patients are introduced to the traditional chanting of healing sounds as well as a self-care program called ' $3-1-2$ '. Those numbers stand for three acupressure points, deep diaphragmatic breathing, and two squats. Healing sounds and '3-1-2' are taught as self-care interventions.

Last but not least, all patients receive nutritional counseling which provides general recommendations in accordance with TCM dietary principles as well as specific guidance tailored to the individual's TCM diagnoses. While our hospital strives to offer nutritious and wholesome meals, and while we make numerous allowances for special dietary needs or preferences, it would be overly complicated to offer each patient a diet tailored specifically to their diagnoses, tastes, and idiosyncrasies (e.g. allergies, intolerances, low carbohydrates, vegetarian, or vegan preferences, etc.). Thus, we cannot fully practice what we preach; patients are encouraged to apply our dietary recommendations after returning home.

\section{Individualised Treatment}

According to the conceptual framework of TCM, the choice of treatments is guided by the general objectives of improving the flow of Qi and of the bodily fluids (micro-circulation) and of regulating the organ networks to improve the vegetative, muscular, and mental functions [1].

Based on the TCM diagnoses the following treatment objectives would be identified for the hypothetical patient with chronic pain and depression:

The finding of liver-Qi stagnation and stasis of the blood calls for a treatment that moves the liver-Qi. The diagnosis of spleen-Qi blockage with spleen-Qi deficiency requires a therapy that moves and strengthens the spleen-Qi. The heart-Qi disturbance, in turn, should be addressed with interventions that move the heart-Qi. Finally, the kidney-Qi deficiency needs to be countered by a strengthening of the kidney-Qi. To these ends, the following Chinese medicinal and herbal remedies and acupuncture points would be prescribed:

In order to move the liver-Q $i$, the patient would take Chinese herbs that have been identified to regulate liver-Qi (e.g., Radix Bupleuri-chaihu/Radix Scutellariae-huangqin/Radix Angelicae sinensis-danggui). The acupoints Liver 3 and Large Intestine 4 would get needled.

To move and strengthen the spleen-Qi, herbs such as Fructus Aurantii-zhishi/Radix Pseudostellariae-taizhishen/Poria-fuling would be prescribed. The acupuncturist would needle the points Spleen 6 and Stomach 36 bilaterally, as well as Conception Vessel 12. 
To counter the stasis of the blood, herbs such as Rhizoma Curcumae longae-jianghuang/Radix Achyranthis bidentatae-niuxi/Radix Paeoniae rubra-chishao would be added to the mixture, and points along the back of the patient would get stimulated by acupuncture.

Finally, in order to sedate the mind and alleviate the heart-Qi disturbance, the medicinal remedy Caulis Polygoni multiflorishouwuteng would be administered and the acupoints Pericardium 6 (bilaterally) and Heart 7 would get needled.

In addition, the patient would practice twice daily a series of 18 exercises derived from the Tai Chi-Qigong traditions (see above). Furthermore, the patient is counseled how to optimize her diet according to Chinese dietary principles in order to support her treatment goals.

Following discharge, the TCM medicinal remedies should be continued at a lower dosage for several months. The patient would be advised to incorporate the Tai Chi-Qigong exercises as well as numerous other dietary and lifestyle recommendations into her daily routine.

The example above serves to illustrate the systematic process that guides the TCM diagnoses and treatments: It is a process of pattern recognition that moves from the diagnostic analysis to the therapeutic application in an organized fashion. In most cases, the primary treatment modalities are the application of medicinal remedies and the stimulation of the meridian system.

\section{Psychotherapeutic and Psychoeducational Programs}

The conceptual framework of the psychological treatment components is behavioral in orientation with an emphasis on (a) improving the regulation of affects, (b) reducing dysfunctional cognitions, and (c) teaching patients to take charge of their healing process through lifestyle adjustments, self-care applications, and preventive measures.

A unique feature of our treatment approach is the utilization of a comprehensive, web-based health-maintenance program [2]. Even prior to admission, patients receive access to this program and are encouraged to take advantage of its many features and components. The data entered by patients provide information for our therapists; simultaneously, they serve as a progress assessment tool for the patients themselves. The data entered by the hospital include diagnostic information (including lab data) and a record of all therapeutic interventions. Thus, the patient has access to self-generated as well as hospital-generated information. In addition, the health maintenance program links to a wealth of in-depth information, offering each patient the opportunity to further explore many health and lifestyle topics that get introduced, and preferably practiced, in our multi-disciplinary presentations and lectures (see below).

The health maintenance program serves as yet another avenue to encourage patients to get informed and actively engaged in their healing process, both during and after the inpatient stay. This computer application is designed to facilitate an interactive learning process which is founded on the didactic concept of 'blended learning.'
Above all, we try to convey a psychosomatic understanding of the mind-body connection. This often necessitates changing the patient's implicit 'disease model' from a largely external to a more internal locus of control. To such end, we draw on Western psychological insights, impart health and lifestyle information, and explain Eastern mind-body concepts. For instance, we may educate our patients about the basic TCM premise that disturbances in the flow of Qi due to internal factors, such as excessive or imbalanced emotions, can lead to the manifestation of functional (Qi-flow disturbances) or somatic symptoms (Qi- and blood-flow disturbances).

\section{Individual Psychotherapy}

Following a first encounter between patient and psychotherapist during the joint intake conference (see above), an in-depth intake interview is conducted on the second day of the inpatient stay. Topics usually addressed are: past and current physical and emotional symptoms; mental status and level of functioning; anamnestic data regarding the family of origin, educational and vocational history, and significant relationships; stressors, resources, and satisfaction in various areas of life. The purpose of the intake interview is, (a) to establish a treatment alliance between patient and therapist; (b) to arrive at a psychopathological ICD diagnosis; and (c) to determine treatment goals that reflect a psychosomatic understanding of the presenting problems.

In a more or less formalized process, patient and therapist jointly develop individualised treatment goals, such as:

- Increased awareness of emotions, cognitions, and inner states;

- Improved self-regulatory competencies, especially the increased ability to relax and reduce stress;

- Improved sense of self; reduced self-critical cognitions; greater kindness toward self;

- Heightened sense of responsibility, agency and control, particularly regarding health, self-care, diet, and prevention;

- Reduced anxiety and avoidance behaviors;

- Improved relationship patterns (e.g., setting limits; being more authentic or assertive);

- Processing of stressful or traumatic life experiences;

- Counseling regarding specific deficits or symptoms (e.g., sleep, pain, exercise, breathing, diet, anxiety, depression);

- Development of life goals, action plans, and/or after-care recommendations;

- Motivating the patient to pursue outpatient (psycho)therapies. In the course of the inpatient stay, the patient takes part in at least one individual psychotherapy session (50-60 min) per week. During the last session, a progress review takes place, often taking into account the data from before/after self-reports (ISR). Most patients receive after-care recommendations and, when appropriate, suggestions regarding further outpatient treatments.

\section{Psychoeducational Programming and Lifestyle Medicine}

Over the span of the inpatient stay, all patients participate in a nine-part, multi-disciplinary group program, conducted in equal parts by Western physicians, psychologists, and health coaches. 
Fig. 2. Comprehensive treatment process at the TCM hospital Bad Kötzting.

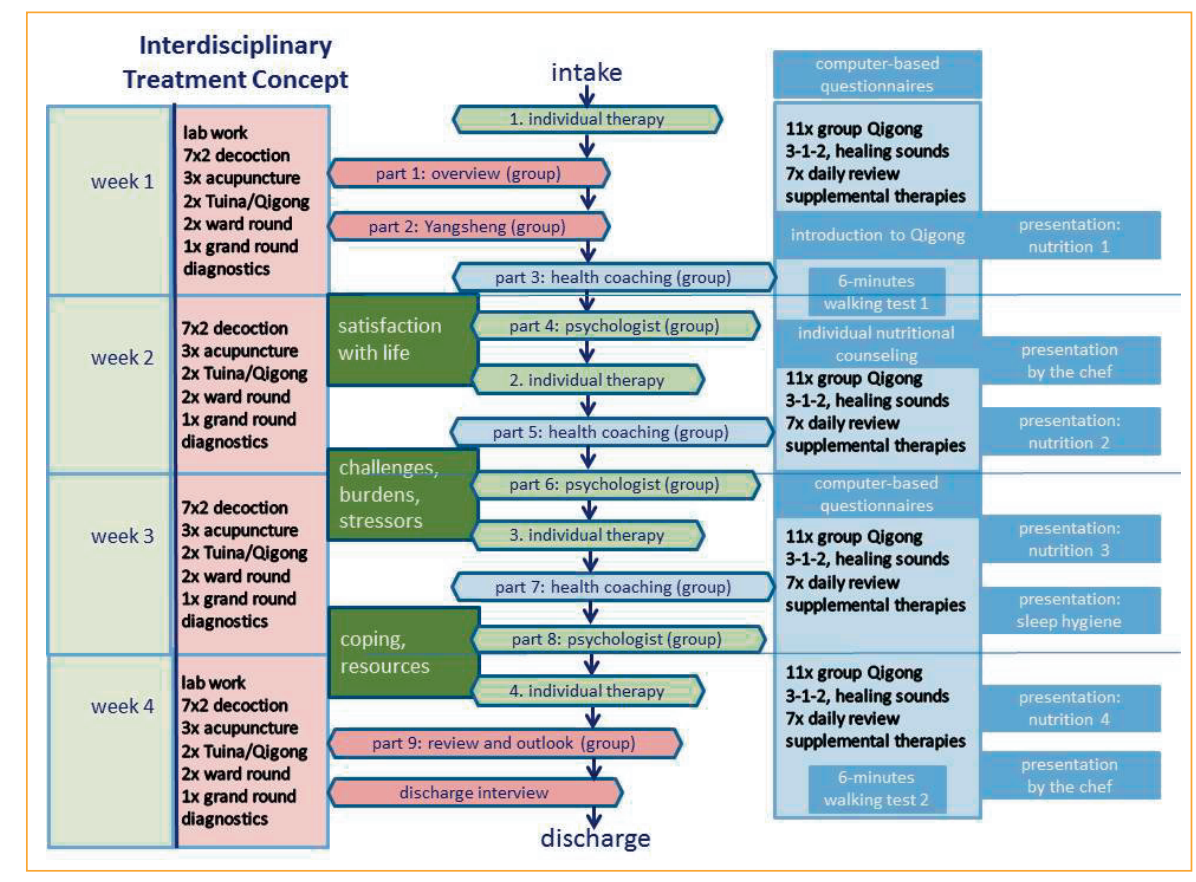

- Part 1 (physician) gives an overview of the treatment program and explains the underlying principles.

- Part 2 (physician) introduces ancient Chinese ideas about Yangsheng (self-care and prevention) and builds a bridge to Western concepts with special reference to data collected through our web-based health maintenance program. The topics discussed pertain to general health factors; satisfaction with various areas of life; affect management; diet; exercise, and relaxation.

- Part 3 (health coach) covers basic concepts of stress and coping in relation to health or a lack thereof.

- Part 4 (psychologist) introduces the concept of emotional intelligence with an emphasis on self-awareness, mindfulness, and the attitude of gratitude as an antidote to bitterness.

- Part 5 (health coach) discusses the significance of emotions for a person's health and strategies for improving affect management.

- Part 6 (psychologist) addresses the effects of chronic and traumatic stress and elaborates on stress-inducing cognitive patterns (be liked, be perfect, be on guard, etc.).

- Part 7 (health coach) discusses resiliency, resources, agency and control, and self-motivation.

- Part 8 (psychologist) reviews cognitive-behavioral strategies for reducing stress-inducing cognitive patterns. In addition, the process of self-governed change is discussed, emphasising responsibility, agency, and resources.

- Part 9 (physician) reviews the treatment program, the takehome messages, and the significance of implementing healthpromoting lifestyle adjustments after the return home.

This mandatory nine-part program outlined above is augmented by several presentations about diet and nutrition, sleep hygiene, and additional lifestyle topics.

\section{The Inpatient Process: Pulling It All Together}

The course of treatment over time is summarized in figure 2 .

In summary, over the past 25 years the treatment program at our hospital has developed into a multimodal approach that synergistically intertwines diverse therapies drawn from Eastern and Western traditions. Combining the best of both approaches, we have found that the total amounts to more than the sum of its parts.

\section{Disclosure Statement}

All the authors are employed at the hospital; A.S. is the owner of the institution.

\section{References}

1 So RWL, Wong HS, Ko KM: A traditional Chinese medicine approach in treating depression by promoting liver qi circulation: a western medicine perspective. Chinese Medicine 2015;6:187-195.
2 Melchart D, Eustachi A, Wellenhofer-Li Y, Bohnes E, Doerfler W: Individual health management - a comprehensive lifestyle counselling program for health promotion, disease prevention and patient education. Forsch Komplementmed 2016;23:30-35. 